\title{
Renal Podocyte Injury in a Rat Model of Type 2 Diabetes Is Prevented by Metformin
}

\author{
Junghyun Kim, Eunjin Shon, Chan-Sik Kim, and Jin Sook Kim \\ Traditional Korean Medicine (TKM) Based Herbal Drug Research Group, Herbal Medicine Research Division, \\ Korea Institute of Oriental Medicine, 1672 Yuseongdaero, Yuseong-gu, Daejeon 305-811, Republic of Korea \\ Correspondence should be addressed to Jin Sook Kim, jskim@kiom.re.kr
}

Received 15 June 2012; Revised 23 August 2012; Accepted 29 August 2012

Academic Editor: Yoshio Shimizu

Copyright ( $(2012$ Junghyun Kim et al. This is an open access article distributed under the Creative Commons Attribution License, which permits unrestricted use, distribution, and reproduction in any medium, provided the original work is properly cited.

Hyperglycemia promotes oxidative stress and hence generation of reactive oxygen species (ROS), which is known to play a crucial role in the pathogenesis of diabetic nephropathy. Metformin, an oral hypoglycemic drug, possesses antioxidant effects. The aim of this paper is to investigate the protective effects of metformin on the injury of renal podocytes in spontaneously diabetic Torii (SDT) rats, a new model for nonobese type 2 diabetes. Metformin $(350 \mathrm{mg} / \mathrm{kg} /$ day $)$ was given to SDT rats for 17 weeks. Blood glucose, glycated haemoglobin (HbA1c), and albuminuria were examined. Kidney histopathology, renal 8-hydroxydeoxyguanosine (8-OHdG) levels and apoptosis were examined. In 43-week-old SDT rats, severe hyperglycemia was developed, and albuminuria was markedly increased. Diabetes induced significant alterations in renal glomerular structure. In addition, urinary and renal 8 OHdG levels were highly increased, and podocyte loss was shown through application of the TUNEL and synaptopodin staining. However, treatment of SDT rats with metformin restored all these renal changes. Our data suggested that diabetes-induced podocyte loss in diabetic nephropathy could be suppressed by the antidiabetes drug, metformin, through the repression of oxidative injury.

\section{Introduction}

Diabetic nephropathy is one of the most serious complications of both type 1 and type 2 diabetis mellitus [1]. The clinical hallmarks of diabetic nephropathy include progressive albuminuria followed by a gradual decline in renal function [2]. The loss of glomerular podocytes precedes and predicts the onset of clinical nephropathy and may be an early pathological manifestation of diabetic nephropathy [3, 4]. Among the three intrinsic cells in glomerulus, podocyte is one of the important ingredients of filtration barrier which has special cytobiological trait and physiological function. The injury of podocyte can inevitablely lead to the occurrence of proteinuria [5]. Patients with advanced diabetic nephropathy have few treatment options and often have a poor prognosis [6].

Metformin has been widely used for treating type 2 diabetes without the stimulation of insulin production [7]. In previous study, metformin could reduce macrovascular morbidity and mortality, suggesting that it achieved its antiatherogenic, anti-inflammatory, and antioxidant effects $[8,9]$. Moreover, metformin significantly decreased the urine albumin excretion rate in patients with type 2 diabetes [10]. The benefits of metformin with regard to the risk of cardiovascular outcomes and metabolic parameters suggest its clinical use in treating chronic kidney disease [11]. The precise mechanisms beyond the effect of metformin on glucose still are obscure. Recent studies suggest that therapeutic effect of metformin might be mediated by its action on AMP-activated kinase (AMPK) in peripheral tissues $[12,13]$. Other studies show that metformin decreases intracellular ROS $[14,15]$.

Diabetic animal models have a critical role in the elucidation of the mechanisms of diabetic complications and the development of novel drugs as treatments. The spontaneously diabetic Torii (SDT) rat is a new model for nonobese type 2 diabetes that spontaneously develops hyperglycemia and glucose intolerance resulting from decreased insulin secretion accompanying $\beta$-cell degeneration [16]. SDT rat is an ideal model for investigating diabetic complications with 
gradual progression and is the only diabetic model known with progression to proliferative retinopathy $[17,18]$.

In the present study, we examined the utility of the SDT rat as a model of diabetic nephropathy, and investigated the preventive effect of metformin on the injury of diabetic glomerular podocytes in SDT rats.

\section{Materials and Methods}

2.1. Animals. The experiments were performed according to the National Institutes of Health (NIH) Guide for the Care and Use of Laboratory Animals and approved by the Korea Institute of Oriental Medicine Institutional Animal Care and Use Committee. Male SDT rats and age-matched SpragueDawley (SD) rats were purchased from CLEA Japan (Tokyo, Japan) and acclimated for 1 week prior to the study. Rats were individually housed in plastic cages and maintained at $24^{\circ} \mathrm{C}$ $\pm 2^{\circ} \mathrm{C}$ with a $12 \mathrm{~h}$ light: dark cycle and received a basal diet (ground Purina rat chow, Ralston Purina, MO, USA) and tap water ad libitum. At 25 weeks of age, the animals were randomly divided into three groups as follows: (1) normal SD rats (NOR, $n=10$ ), (2) vehicle-treated SDT rats (SDT, $n=10$ ), and (3) SDT rats treated with metformin (SDT + MET, $350 \mathrm{mg} / \mathrm{kg}$ body weight). Metformin was administered once a day orally for 17 weeks. The blood glucose level and body weight were monitored consecutively, and glycated hemoglobin was determined by a commercial kit (Unimate HbAlc, Roche Diagnostics, Mannheim, Germany).

2.2. Metabolic and Morphological Analysis. When the rats reached 43 weeks of age, blood glucose and HbAlc (A1C) were measured using an automated analyzer (Wako, Japan). Blood samples were collected from the tail vein after a $16 \mathrm{~h}$ fast. Individual rats were placed in metabolic cages to obtain $24 \mathrm{~h}$ urine collections, and daily urinary albumin excretion levels were measured. Renal cortexes were fixed in $10 \%$ formaldehyde and embedded in paraffin, and $4 \mu \mathrm{m}$ thick sections were prepared. The sections were stained with periodic acid-Schiff (PAS) reagent or Masson's modified trichrome to assess glomerulosclerosis and demonstrate collagenous tubulointerstitial matrix, respectively.

2.3. Measurement of Urinary 8-Hydroydeoyguanosine (8OhdG). $24 \mathrm{~h}$ urine samples were collected from rats. The samples were purged of air with a steam of nitrogen to prevent the artificial formation of $8-\mathrm{OHdG}$ and stored frozen at $-80^{\circ} \mathrm{C}$ until analyzed. Urine samples were centrifuged at 2,000 $\mathrm{g}$ for $20 \mathrm{~min}$, and after proper dilution, the supernatant was used for the determination of 8-OHdG by a competitive enzyme-linked immunosorbent assay (ELISA) kit (Japan Institute for the Control of Aging, Fukuroi, Japan). The urinary 8-OHdG was expressed as total amounts excreted in $24 \mathrm{~h}$.

2.4. Measurement of 8-OHdG Levels in Renal Tissues. The kidney was rapidly excised. The renal cortex tissues were homogenized in $5 \mathrm{~mL}$ of $50 \mathrm{mmol} / \mathrm{L}$ Tris-HCl $(\mathrm{pH} \mathrm{7.4).}$ The homogenates were centrifuged at $800 \mathrm{~g}$ for $10 \mathrm{~min}$ to precipitate nuclear fraction, and $1 \mathrm{~mL}$ of solution containing $10 \mathrm{mmol} / \mathrm{L}$ EDTA, $10 \mathrm{mmol} / \mathrm{L}$ Tris- $\mathrm{HCl}(\mathrm{pH} 8.0)$, $150 \mathrm{mmol} / \mathrm{L} \mathrm{NaCl}$, and $0.2 \%$ SDS was added. After homogenization, the mixture was incubated at $56^{\circ} \mathrm{C}$ for $70 \mathrm{~min}$ with proteinase $\mathrm{K}(700 \mu \mathrm{g} / \mathrm{mL})$ and then heated at $95^{\circ} \mathrm{C}$ for $10 \mathrm{~min}$. DNA was extracted with equal volumes of phenol, chloroform, and isoamyl alcohol $(25: 24: 1)$ and then with chloroform. DNA was precipitated with $70 \%$ ethanol at $-20^{\circ} \mathrm{C}$ for $2 \mathrm{~h}$. DNA was resuspended in $10 \mathrm{mmol} / \mathrm{L}$ Tris$\mathrm{HCl}$ and $0.1 \mathrm{mmol} / \mathrm{L}$ EDTA ( $\mathrm{pH}$ 8.0). Five microliters of $200 \mathrm{mmol} / \mathrm{L}$ sodium acetate buffer $(\mathrm{pH} 4.8)$ and $5 \mu \mathrm{g}$ nuclease P1 (Invitrogen, CA, USA) were added to $45 \mu \mathrm{L}$ DNA samples. The mixtures were incubated at $37^{\circ} \mathrm{C}$ for $1 \mathrm{~h}$ to digest the DNA to nucleotides. Then, $5 \mu \mathrm{L}$ of $500 \mathrm{mmol} / \mathrm{L}$ Tris- $\mathrm{HCl}(\mathrm{pH} 8.0), 10 \mathrm{mmol} / \mathrm{L} \mathrm{MgCl}_{2}$, and 0.6 units alkaline phosphatase (Toyobo, Osaka, Japan) were added to the samples. The mixtures were incubated at $37^{\circ} \mathrm{C}$ for $1 \mathrm{~h}$ to hydrolyze the nucleotides to nucleosides. The nucleoside samples were used for the determination of 8 -OHdG by competitive ELISA kit as described above.

2.5. Double Labelling for TUNEL and Synaptopodin Expression. TUNEL was performed with the In-situ cell death detection kit AP (Roche Diagnostics, Mannheim, Germany) according to the manufacturer's instructions. Apoptotic cells were detected with a colour solution containing nitroblue tetrazolium (NBT, Roche Diagnostics) and 5-bromo4-chloro-3-indolylphosphate (BCIP, Roche Diagnostics). Labelling with mouse anti-synaptopodin antibody (Santa Cruz Biotechnology, CA, USA) was performed subsequently on the same sections with secondary detection by horseradish peroxidase-conjugated anti-mouse IgG antibody (Santa Cruz) and an AEC Red peroxidase substrate kit (Vector Laboratories, Burlingame, California). To prevent crossreaction between the two labelling procedures the slides were incubated with normal mouse serum (Dako) after the TUNEL labelling. For morphometric analysis, the areas of positive signal for synaptopodin per glomerulus over a total of 40 glomeruli was determined using Image J software, and cells dually labelled for TUNEL and synaptopodin were counted.

2.6. Immunohistochemical Staining for WT-1. Staining was performed as previously described [19]. Antibody was rabbit anti-Wilms tumor antigen-1 (WT-1, 1:250, Santa Cruz). The sections were visualized by nitroblue tetrazolium chloride (NBT) and 5-bromo-4-chloro-3-indolyl phosphate (BCIP) substrate staining and counterstained with methyl green. Negative controls for immunohistochemistry were run by incubating the sections with nonimmune serum instead of the primary antibody. For morphometric analysis, the positive cell numbers per glomerulus in a total of 40 glomerulus was determined.

2.7. Western Blotting Analysis in Retinal Tissues. Proteins were extracted from renal tissues, and then $20 \mu \mathrm{g}$ of protein lysates was separated by SDS-polyacrylamide gel electrophoresis and transferred to nitrocellulose membranes (Biorad, CA, USA). Membrane was probed with mouse 
TABLE 1: Metabolic and physical parameters.

\begin{tabular}{lccc}
\hline & NOR & SDT & MET \\
\hline Body weight $(\mathrm{g})$ & $713.9 \pm 29.7$ & $375.5 \pm 52.4^{*}$ & $430.5 \pm 13.4$ \\
Blood glucose $(\mathrm{mg} / \mathrm{dL})$ & $144.1 \pm 21.0$ & $419.2 \pm 21.2^{*}$ & $359.5 \pm 52.3$ \\
HbA1c $(\%)$ & $3.49 \pm 0.07$ & $9.13 \pm 0.37^{*}$ & $7.82 \pm 0.29$ \\
\hline
\end{tabular}

NOR: normal rat; SDT: spontaneously diabetic Torii rat; MET: SDT rat treated with metformin $(350 \mathrm{mg} / \mathrm{kg} /$ day $)$. All data are expressed as mean \pm SE $(n=8) .{ }^{*} P<0.01$ versus NOR group. ${ }^{\#} P<0.01$ versus SDT group.

anti-AMPK antibody (Santa Cruz) and mouse anti-phospho-AMPK antibody (Santa Cruz), and then the immune complexes were visualized with an enhanced chemiluminescence detection system (Amersham Bioscience, NJ, USA).

2.8. Statistical Analysis. Data are expressed as mean $\pm \mathrm{SE}$ and analyzed by one-way analysis of variance (ANOVA) followed by Tukey's multiple comparison test or by unpaired Student's $t$-test using GraphPad Prism 5.0 software (Graph pad, San Diego, CA, USA). Differences with a value of $P<0.01$ were considered statistically significant.

\section{Results}

3.1. Body Weight and Metabolic Parameters in Blood. In SDT diabetic rats at 43 weeks of age, body weight was decreased compared with normal rats and did not change compared with rats that treated metformin. Blood glucose and HbAlc levels were significantly increased in SDT rats $(P<0.01$ versus normal rats). Metformin induced a minor decrease of blood glucose and HbA1c levels (Table 1).

3.2. Morphology and Renal Function. Mesangial matrix expansion is considered a hallmark of diabetic nephropathy. At 43 weeks of age, SDT rats showed focal mesangial matrix expansion, tubulointerstitial damage, and albuminuria were significantly increased in SDT rats compared to normal rats. Metformin treatment ameliorated mesangial expansion and albuminuria, compared with untreated SDT rats (Figures $1(\mathrm{a})$ to $1(\mathrm{c}))$.

3.3. Quantification of 8-OHdG in Urine and Renal Tissue. The total amounts of urinary 8-OHdG excretion were significantly greater in SDT rats than in normal rats (Figure 2(a)). The levels of 8-OHdG in renal cortex tissues were significantly increased in SDT rats as compared with those from the normal rats (Figure 2(b)). Similarly, There was increased expression of $8-\mathrm{OHdG}$ in the podocytes of SDT rats and this labelling had a nuclear and/or perinuclear localization (Figure 2(c)). Metformin reduced these diabetesinduced increases in urinary and renal 8-OHdG.

3.4. Apoptosis of Renal Podocytes in SDT Rats. In SDT rats, many podocytes, mesangial cells, and capillary endothelial cells were positively labelled by the TUNEL technique. However, metformin prevented the increase in the positive cells that was seen in normal kidney. Average numbers of podocytes per glomerular section were determined by counting cells and measuring areas that were positively labeled with two podocyte markers, such as synaptopodin and WT1 In SDT rats, synaptopodin and WT-1-positive cell counts tended to decrease compared with age-matched normal rats. Treatment with metformin visibly increased the positive cells and areas in the kidney glomeruli (Figures 3(a) to 3(d)). In dual-labelled sections, TUNEL-positive cells were localized to the regions of synaptopodin expression consistent with apoptosis of podocytes in diabetic renal tissue (Figures 3(a) and $3(\mathrm{e}))$.

3.5. Changes in AMPK Activation in SDT Rats. We assessed whether metformin activates AMPK in the kidney using immunoblotting for phosphorylation of AMPK. Activation of AMPK was significantly reduced in the kidney of SDT rats compared with normal rats, and metformin induced AMPK activation in the kidney (Figures 4(a) and 4(b)).

\section{Discussion}

Animal models traditionally employed in the investigation of diabetic nephropathy include STZ-induced diabetic rats, but the general pathophysiology of disease in these models differs from that of NIDDM in man. The SDT rat, a model of nonobese type 2 diabetes, was established in 1997 by Shinohara et al. [18]. Hyperglycemia spontaneously develops in SDT rats, predominantly due to an insulin secretary defect. In addition to marked hyperglycemia, renal complications are a characteristic of SDT rats [20]. Based on the findings of the present study, SDT rat may be an appropriate an animal model for investigation of diabetic nephropathy. This model has many similarities to human NIDDM and the development of diabetes mellitus in SDT rats is accompanied by functional and morphological kidney damage that resembles human diabetic nephropathy $[20,21]$.

Growing evidence suggests that the overproduction of ROS may be the key initiating event that leads to the longterm development of diabetic complications [22]. However, the specific mechanisms that link hyperglycemia with oxidative stress and diabetic nephropathy are poorly understood. In general, oxidative stress can affect nucleic acids and generate various modified bases in DNA. $8-\mathrm{OHdG}$ is the most abundant and appears to play a crucial role in mutagenesis [23]. Oxidation of guanine to form 8-OHdG acts as a marker of oxidative DNA damage [24]. When DNA is damaged, cells initiate a response, such as DNA repair, cellcycle delay, or induction of apoptosis [25]. Increased levels of 8-OHdG have been reported in urine [26], mononuclear cells [27] and skeletal muscles [28] of diabetic patients. The levels of 8-OHdG were increased in kidney tissues of STZinduced diabetic rats [29]. In the present study, we further revealed that levels of $8-\mathrm{OHdG}$ were increased in renal tissues of SDT rats. To localize expression of $8-\mathrm{OHdG}$ in diabetic kidneys, kidney tissues were analyzed by imunohistochemical staining. Although the method for 8-OHdG detection stains nuclear DNA as well as mitochondrial DNA (mtDNA), the staining was localized mainly in the cytoplasm, indicating that this oxidative adduct was present in mtDNA, but not in nuclear DNA. It is accepted that vulnerability to oxidative damage and subsequent mutations is $10-20$ times greater 

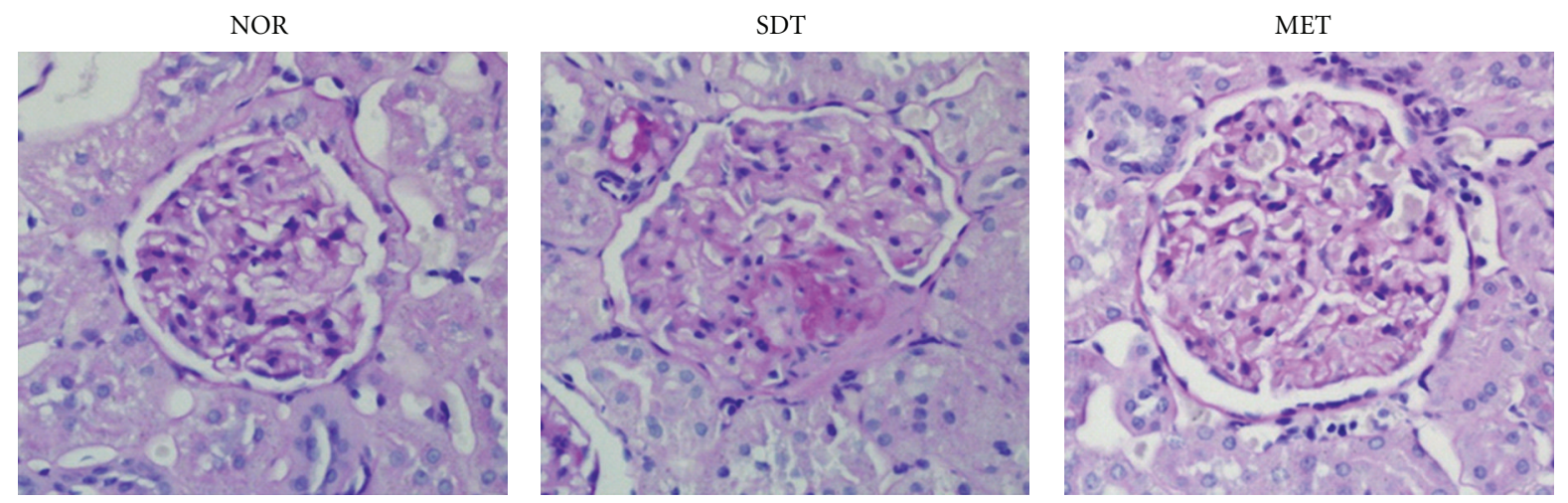

(a)
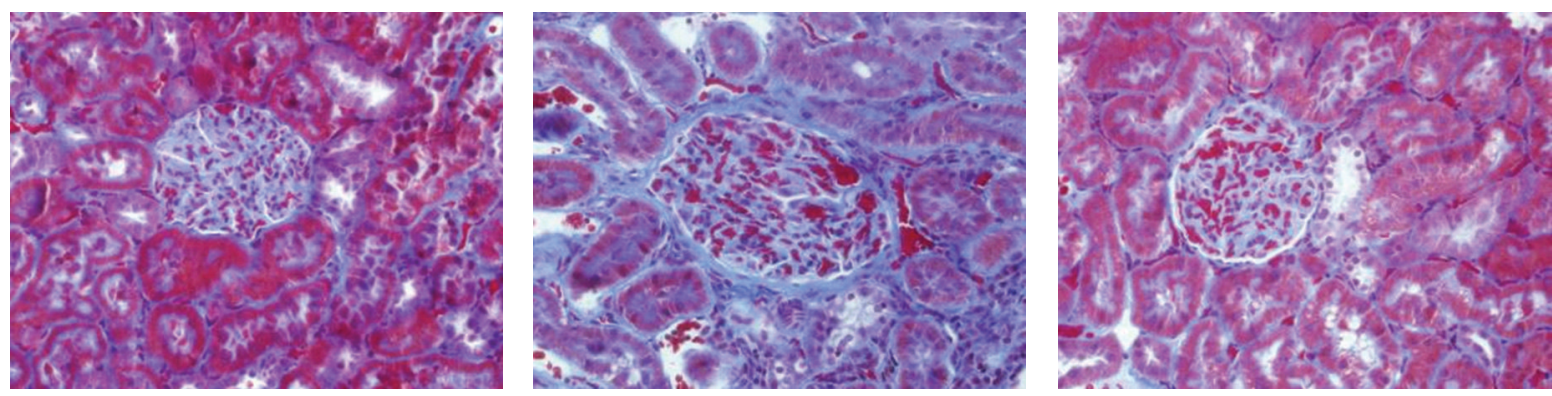

(b)

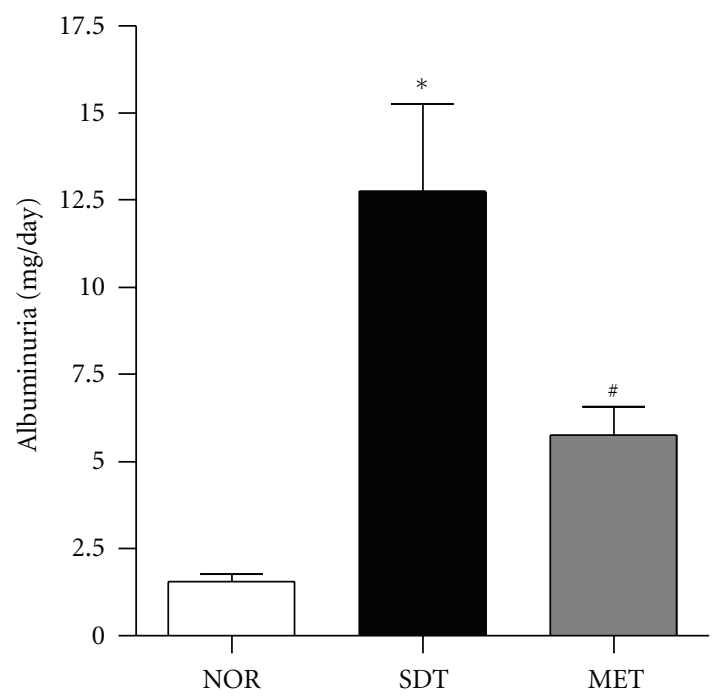

(c)

Figure 1: Renal histopathology and function(a). Periodic acid-Schiff staining of glomeruli, $\times 400$ magnification (b). Masson's trichrome stained renal sections, $\times 400$ magnification (c). Albuminuria in normal rat (NOR), spontaneously diabetic Torii rat (SDT) and SDT rat treated with $350 \mathrm{mg} / \mathrm{kg}$ metformin (MET). All data are expressed as mean $\pm \mathrm{SE}(n=8)$. ${ }^{*} P<0.01$ versus NOR group, ${ }^{\#} P<0.01$ versus SDT group.

for mtDNA than for nuclear DNA [30, 31]. In parallel with the results of renal tissues, the amounts of urinary 8-OHdG excretion were markedly increased in SDT rats. Although urinary 8-OHdG levels are supposed to be markers of the total systemic oxidative stress in vivo [32], the relative contribution of renal 8-OHdG to urinary 8-OHdG should be evaluated in future studies.
The generation of ROS through oxidative stress causes cell death [33]. Apoptosis, which is characterized by chromatin condensation, DNA fragmentation, and the activation of caspases, has been implicated in the pathogenesis of various renal diseases, including diabetic nephropathy [34, 35]. ROS are potent inducers of apoptosis in various cell types including murine and human podocytes [36-38]. Our 


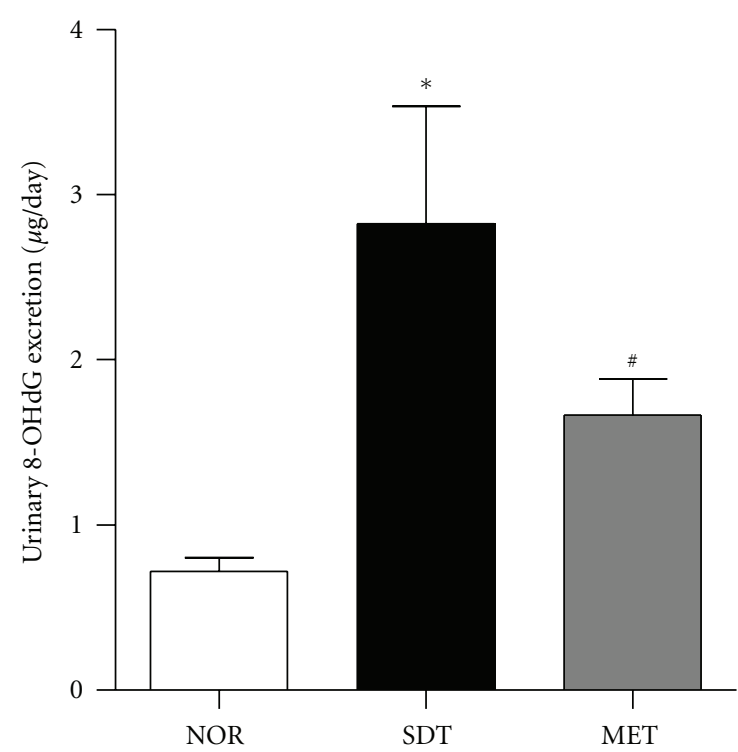

(a)

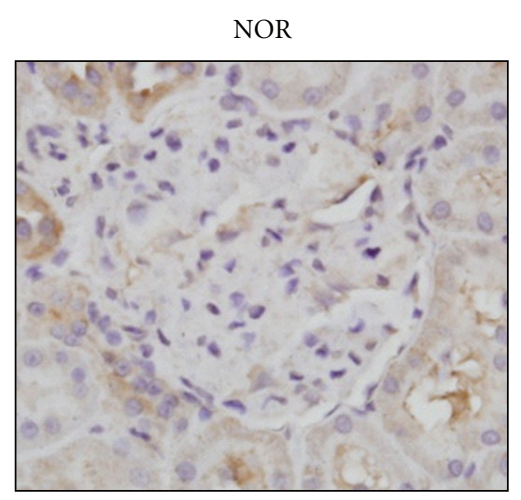

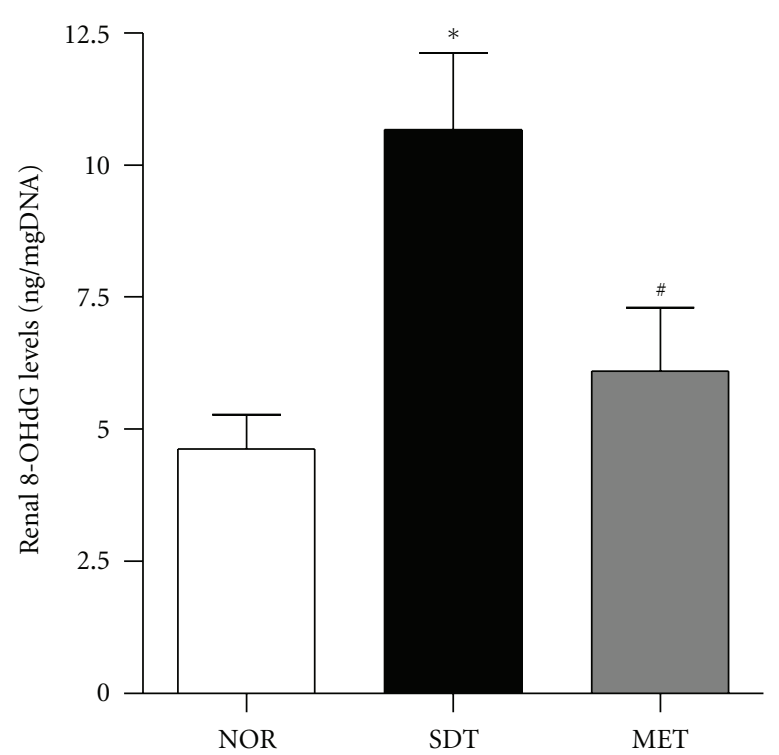

(b)
SDT

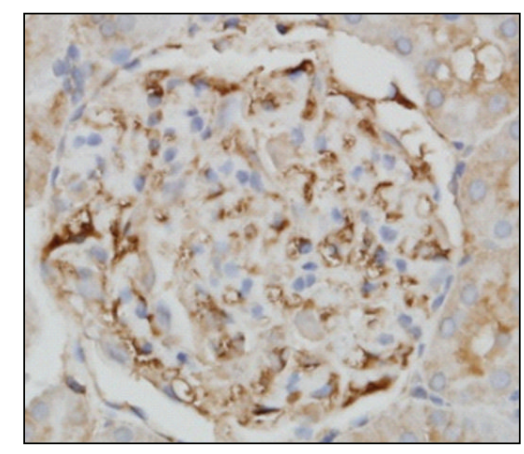

(c)

FIgURE 2: Expression of 8-OHdG in urine and renal tissues. (a) Uriany 8-OHdG excretion levels, (b) renal 8-OHdG levels, and (c) immunohistochemical stating for 8-OHdG in normal rat (NOR), spontaneously diabetic Torii rat (SDT) and SDT rat treated with $350 \mathrm{mg} / \mathrm{kg}$ metformin (MET). All data are expressed as mean $\pm \mathrm{SE}(n=8) .{ }^{*} P<0.01$ versus NOR group, ${ }^{\#} P<0.01$, versus SDT group.

present study shows that the density of podocytes decreases in SDT rats in association with increased albuminuria. Podocyte apoptosis has been demonstrated to correlate with worsening albuminuria [38]. Furthermore, strong evidence has established a role for intracellular ROS as potent inducers of podocyte apoptosis [39]. The increase in antioxidant enzymes such as heme oxygenase-1 has been shown to reduce podocyte apoptosis under diabetic conditions [40]. Consistent with this interpretation, the results of the present study demonstrate that the increased 8-OHdG concentration in affected renal glomeruli is involved, at least in part, in the injury of podocytes.

In addition, we examined the effect of intervention by metformin treatment on the increases in 8-OHdG levels and podocyte loss in kidney tissues of diabetes. It is of great interest that intervention by metformin treatment inhibited both increases in renal 8-OHdG levels and podocyte loss in kidney of SDT rats. The treatment of SDT rats with metformin slightly decreased blood glucose and HbAlc levels.
Hyperglycemia, a key clinical manifestation of diabetes, is supposed to generate ROS through various mechanisms, such as increased formation of advanced glycation end products (AGEs) [41], enhanced polyol pathway [42], increased superoxide release from mitochondria [43], and activation of $\mathrm{NAD}(\mathrm{P}) \mathrm{H}$ oxidase [44]. In our present study, metformin induced only a minor decrease of levels of blood glucose $(22 \%)$ and HbAlc $(23 \%)$ in SDT rats. Similarly, metformin failed to reduce blood glucose levels in ZDF rats [45]. Although metformin has a weak glucose-lowering effect in SDT rats, metformin has significant effects on any parameters of renal structure and function without the strong reduction of blood glucose. The level of albuminuria was decreased by $63 \%$ in metformin-treated SDT rats. However, Fujii $\mathrm{H}$ et al. showed that the treatment of insulin decreased the level of HbAlc by almost 55\% [46]. The level of albuminuria was also decreased by $62 \%$ in insulin-treated SDT rats. These findings suggest that even in hyperglycemia, it is possible to attenuate podocyte injury by metformin. 

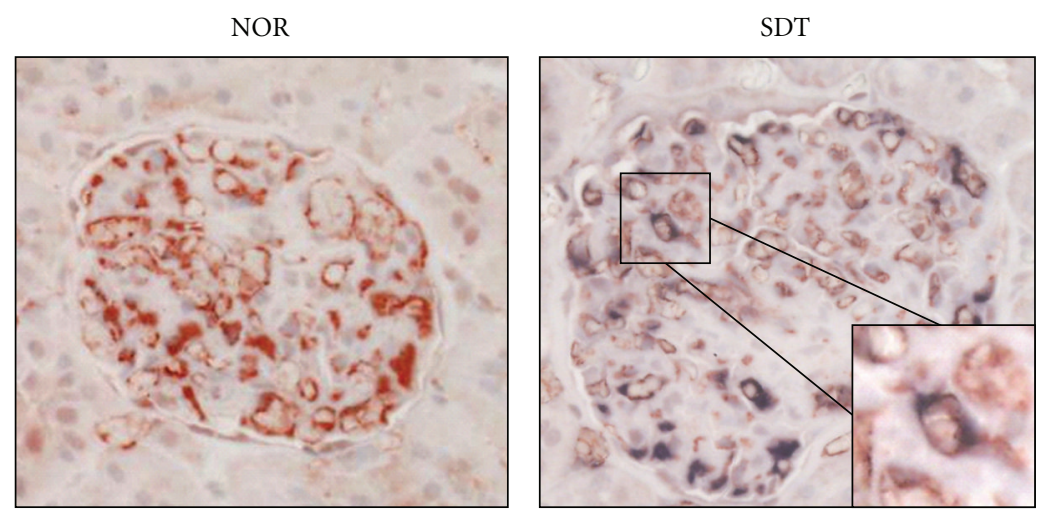

(a)
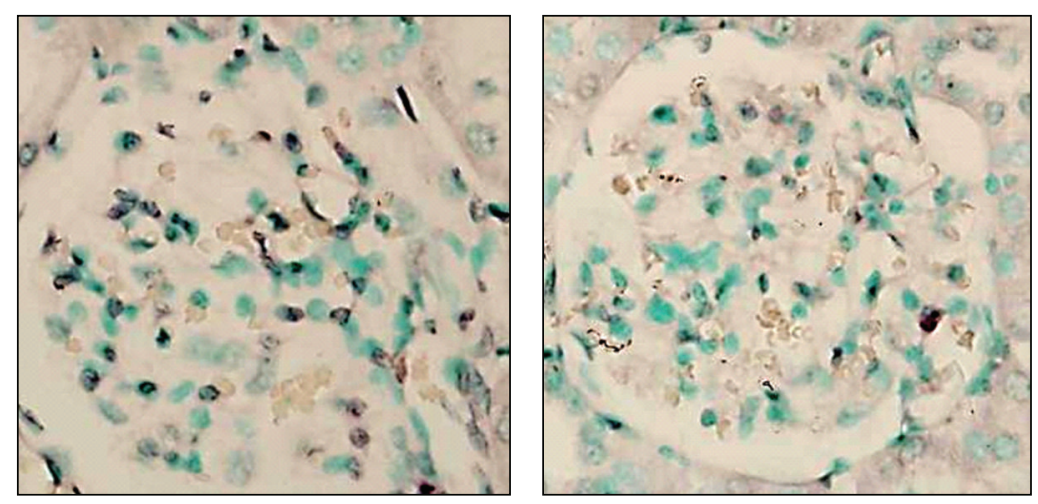

(b)

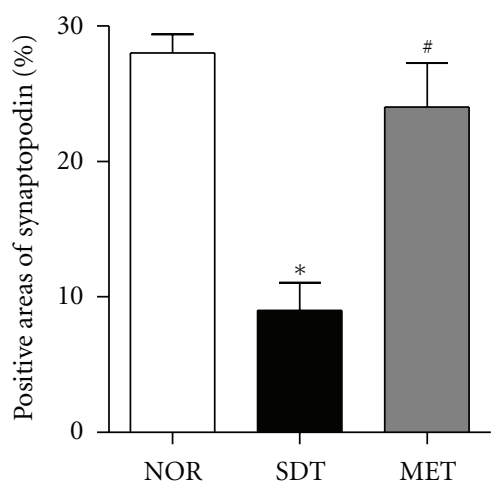

(c)

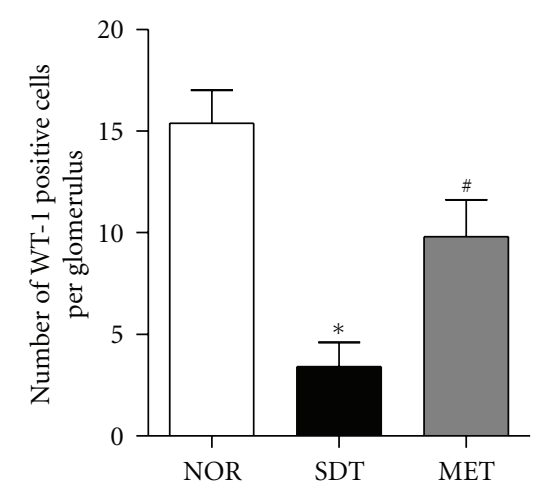

(d)
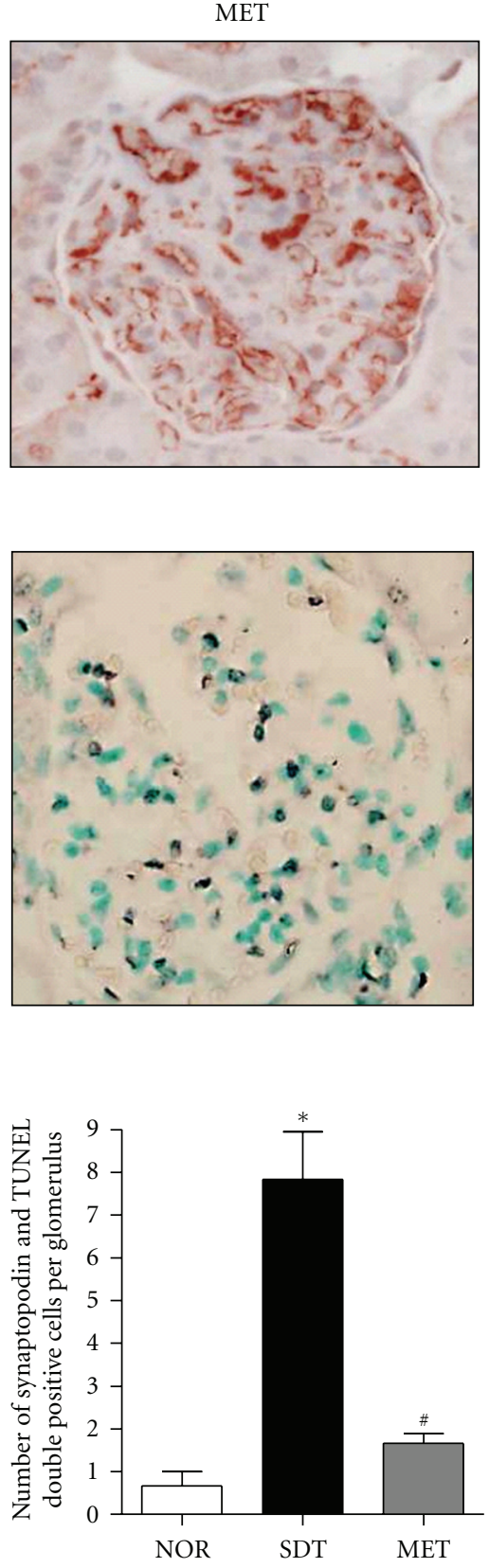

(e)

FIGURE 3: Podocyte loss. A representative photomicrograph of (a) dual labelling for TUNEL (black) and synaptopodin (red) and (b) WT1 (black). $\times 400$ magnification. Double-positive cells (magnified inset) indicates the colocalization of the podocyte marker and apoptosis. Quantitative analyses of (c) positive areas of synaptopodin, (d) positive cells of WT-1 and (e) TUNEL and synaptopodin-double-positive cells. All data are expressed as mean $\pm \mathrm{SE}(n=8) .{ }^{*} P<0.01$ versus NOR group, ${ }^{\#} P<0.01$, versus SDT group.

Metformin has been found to activate AMPK, a major cellular regulator of lipid and glucose metabolism [13]. Previous studies also have shown that metformin is an inhibitor of complex I of the mitochondrial respiratory chain independent of the AMPK pathway $[47,48]$ and a potent inhibitor of AGE formation [49]. AMPK pathway acts as a signal for ATP generation, a process coupled to increase in ROS production. AMPK pathway can be activated by intracellular ROS and reduces ROS levels $[50,51]$. The ability of AMPK to simultaneously reduce ROS levels and counterbalance the overproduction of ROS is an important mechanism for controlling the redox balance during energy production. In present study, we found that the phosphorylation of AMPK was reduced in the kidney of SDT rats, and metformin could restore its alteration. Therefore, metformin is likely exerting some of its effects via improvement of renal oxidative stress. It could be reasonable to assume that the renoprotective effect of metformin might be at least partly, attributed to its 


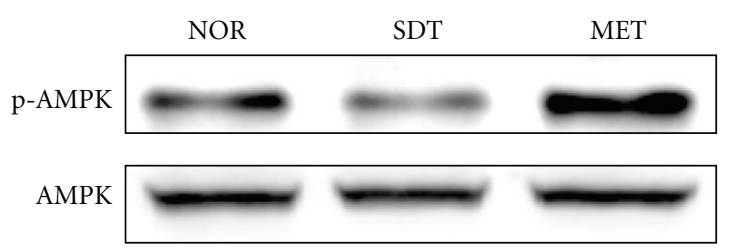

(a)

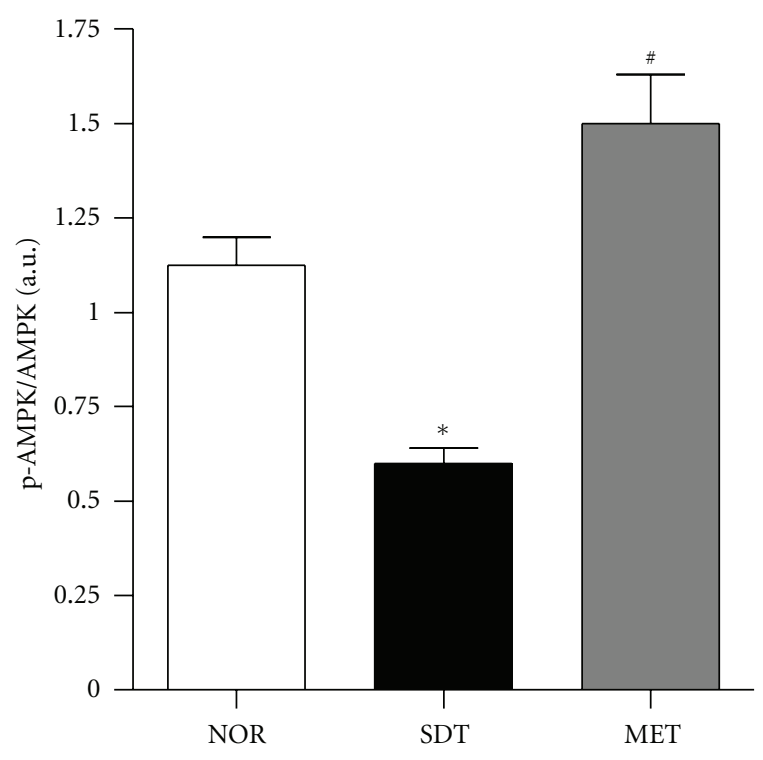

(b)

FIGURE 4: AMPK activation in renal tissues. (a) Representative immunoblots of phospho-AMPK and AMPK in protein extracts from the kidneys of rats of each group. (b) Quantitative analysis of protein expression. All data are expressed as mean \pm SE $(n=8)$. ${ }^{*} P<0.01$ versus NOR group, ${ }^{\#} P<0.01$, versus SDT group.

influence on AGEs formation and ROS production, besides its antihyperglycemic effect. These observations suggest a potential clinical use of metformin in the prevention of diabetic nephropathy by inhibition of AGEs and improving the free-radical defense system. Liu et al. have pointed the beneficial antioxidant effects of metformin in STZ-induced DM in rats [52].

In conclusion, SDT rat is a useful model for the evaluation of the effects of drugs and the investigation of diabetic nephropathy. We also demonstrated that metformin protects against diabetic nephropathy by restoring the biochemical alterations and modulation of oxidative stress, and hence suggests a potential clinical use of metformin in the prevention of diabetic nephropathy.

\section{Conflict of Interests}

The authors declare that there is no conflict of interests.

\section{Acknowledgment}

This research was supported by a Grant [K12040] from the Korea Institute of Oriental Medicine (KIOM).

\section{References}

[1] K. E. White and R. W. Bilous, "Type 2 diabetic patients with nephropathy show structural-functional relationships that are similar to type 1 disease," Journal of the American Society of Nephrology, vol. 11, no. 9, pp. 1667-1673, 2000.

[2] S. M. Mauer, M. W. Steffes, and E. N. Ellis, "Structural-functional relationships in diabetic nephropathy," Journal of Clinical Investigation, vol. 74, no. 4, pp. 1143-1155, 1984.

[3] M. E. Pagtalunan, P. L. Miller, S. Jumping-Eagle et al., "Podocyte loss and progressive glomerular injury in type II diabetes," Journal of Clinical Investigation, vol. 99, no. 2, pp. 342-348, 1997.

[4] T. W. Meyer, P. H. Bennett, and R. G. Nelson, "Podocyte number predicts long-term urinary albumin excretion in Pima Indians with type II diabetes and microalbuminuria," Diabetologia, vol. 42, no. 11, pp. 1341-1344, 1999.

[5] Y. Zhang, B. Chen, X. H. Hou et al., "Effects of mycophenolate mofetil, valsartan and their combined therapy on preventing podocyte loss in early stage of diabetic nephropathy in rats," Chinese Medical Journal, vol. 120, no. 11, pp. 988-995, 2007.

[6] S. Rudberg, B. Persson, and G. Dahlquist, "Increased glomerular filtration rate as a predictor of diabetic nephropathy-an 8 -year prospective study," Kidney International, vol. 41, no. 4, pp. 822-828, 1992.

[7] C. J. Bailey and R. C. Turner, "Metformin," New England Journal of Medicine, vol. 334, no. 9, pp. 574-579, 1996.

[8] UK Prospective Diabetes Study (UKPDS) Group, "Effect of intensive blood-glucose control with metformin on complications in overweight patients with type 2 diabetes (UKPDS 34)," The Lancet, vol. 352, no. 9131, pp. 854-865, 1998.

[9] F. Abbasi, J. W. Chu, T. McLaughlin, C. Lamendola, E. T. Leary, and G. M. Reaven, "Effect of metformin treatment on multiple cardiovascular disease risk factors in patients with type 2 diabetes mellitus," Metabolism, vol. 53, no. 2, pp. 159164, 2004.

[10] N. Amador-Licona, J. M. Guízar-Mendoza, E. Vargas, G. Sánchez-Camargo, and L. Zamora-Mata, "The short-term effect of a switch from glybenclamide to metformin on blood pressure and microalbuminuria in patients with type 2 diabetes mellitus," Archives of Medical Research, vol. 31, no. 6, pp. 571-575, 2000.

[11] H. L. Pilmore, "Review: metformin: potential benefits and use in chronic kidney disease," Nephrology, vol. 15, no. 4, pp. 412$418,2010$.

[12] R. J. Shaw, K. A. Lamia, D. Vasquez et al., "Medicine: the kinase LKB1 mediates glucose homeostasis in liver and therapeutic effects of metformin," Science, vol. 310, no. 5754, pp. 16421646, 2005.

[13] G. Zhou, R. Myers, Y. Li et al., "Role of AMP-activated protein kinase in mechanism of metformin action," Journal of Clinical Investigation, vol. 108, no. 8, pp. 1167-1174, 2001.

[14] A. Gallo, G. Ceolotto, P. Pinton et al., "Metformin prevents glucose-induced protein kinase C- $\beta 2$ activation in human umbilical vein endothelial cells through an antioxidant mechanism," Diabetes, vol. 54, no. 4, pp. 1123-1131, 2005.

[15] N. Ouslimani, J. Peynet, D. Bonnefont-Rousselot, P. Thérond, A. Legrand, and J. L. Beaudeux, "Metformin decreases intracellular production of reactive oxygen species in aortic endothelial cells," Metabolism, vol. 54, no. 6, pp. 829-834, 2005.

[16] T. Sasase, N. Ohta, K. Ogawa et al., "Preventive effects of glycaemic control on ocular complications of Spontaneously Diabetic Torii rat," Diabetes, Obesity and Metabolism, vol. 8, no. 5, pp. 501-507, 2006. 
[17] T. Masuyama, K. Komeda, A. Hara et al., "Chronological characterization of diabetes development in male Spontaneously Diabetic Torii rats," Biochemical and Biophysical Research Communications, vol. 314, no. 3, pp. 870-877, 2004.

[18] M. Shinohara, T. Masuyama, T. Shoda et al., "A new spontaneously diabetic non-obese torii rat strain with severe ocular complications," International Journal of Experimental Diabetes Research, vol. 1, no. 2, pp. 89-100, 2000.

[19] E. J. Sohn, C. S. Kim, Y. S. Kim et al., "Effects of magnolol (5,5'-diallyl-2,2'-dihydroxybiphenyl) on diabetic nephropathy in type 2 diabetic Goto-Kakizaki rats," Life Sciences, vol. 80, no. 5, pp. 468-475, 2007.

[20] T. Ohta, K. Matsui, K. Miyajima et al., "Effect of insulin therapy or renal changes in spontaneously diabetic Torii rats," Experimental Animals, vol. 56, no. 5, pp. 355-362, 2007.

[21] K. Matsui, T. Ohta, T. Oda et al., "Diabetes-associated complications in Spontaneously Diabetic Torii fatty rats," Experimental Animals, vol. 57, no. 2, pp. 111-121, 2008.

[22] P. Rösen, P. P. Nawroth, G. King, W. Möller, H. J. Tritschler, and L. Packer, "The role of oxidative stress in the onset and progression of diabetes and its complications: a summary of a congress series sponsored by UNESCO-MCBN, the American diabetes association and the German diabetes society," Diabetes/Metabolism Research and Reviews, vol. 17, no. 3, pp. 189-212, 2001.

[23] Y. Kuchino, F. Mori, and H. Kasai, "Misreading of DNA templates containing 8-hydroxydeoxyguanosine at the modified base and at adjacent residues," Nature, vol. 327, no. 6117, pp. 77-79, 1987.

[24] K. B. Beckman and B. N. Ames, "Oxidative decay of DNA," The Journal of Biological Chemistry, vol. 272, no. 32, pp. 1963319636, 1997.

[25] O. Zgheib, Y. Huyen, R. A. DiTullio Jr. et al., "ATM signaling and 53BP1," Radiotherapy and Oncology, vol. 76, no. 2, pp. 119-122, 2005.

[26] J. Leinonen, T. Lehtimäki, S. Toyokuni et al., "New biomarker evidence of oxidative DNA damage in patients with noninsulin-dependent diabetes mellitus," FEBS Letters, vol. 417, no. 1, pp. 150-152, 1997.

[27] P. Dandona, K. Thusu, S. Cook et al., "Oxidative damage to DNA in diabetes mellitus," The Lancet, vol. 347, no. 8999, pp. 444-445, 1996.

[28] S. Suzuki, Y. Hinokio, K. Komatu et al., "Oxidative damage to mitochondrial DNA and its relationship to diabetic complications," Diabetes Research and Clinical Practice, vol. 45, no. 2-3, pp. 161-168, 1999.

[29] H. Ha, C. Kim, Y. Son, M. H. Chung, and K. H. Kim, "DNA damage in the kidneys of diabetic rats exhibiting microalbuminuria," Free Radical Biology and Medicine, vol. 16, no. 2, pp. 271-274, 1994.

[30] C. G. Fraga, M. K. Shigenaga, J. W. Park, P. Degan, and B. N. Ames, "Oxidative damage to DNA during aging: 8-Hydroxy2 '-deoxyguanosine in rat organ DNA and urine," Proceedings of the National Academy of Sciences of the United States of America, vol. 87, no. 12, pp. 4533-4537, 1990.

[31] D. A. Merriwether, A. G. Clark, S. W. Ballinger et al., "The structure of human mitochondrial DNA variation," Journal of Molecular Evolution, vol. 33, no. 6, pp. 543-555, 1991.

[32] M. K. Shigenaga, C. J. Gimeno, and B. N. Ames, "Urinary 8hydroxy-2'-deoxyguanosine as a biological marker of in vivo oxidative DNA damage," Proceedings of the National Academy of Sciences of the United States of America, vol. 86, no. 24, pp. 9697-9701, 1989.
[33] A. J. Hale, C. A. Smith, L. C. Sutherland et al., "Apoptosis: molecular regulation of cell death," European Journal of Biochemistry, vol. 236, no. 1, pp. 1-26, 1996.

[34] M. Dalla Vestra, A. Saller, M. Mauer, and P. Fioretto, "Role of mesangial expansion in the pathogenesis of diabetic nephropathy," Journal of Nephrology, vol. 14, no. 4, pp. S51-S57, 2001.

[35] T. Khera, J. Martin, S. Riley, R. Steadman, and A. O. Phillips, "Glucose enhances mesangial cell apoptosis," Laboratory Investigation, vol. 86, no. 6, pp. 566-577, 2006.

[36] F. N. Ziyadeh and G. Wolf, "Pathogenesis of the podocytopathy and proteinuria in diabetic glomerulopathy," Current Diabetes Reviews, vol. 4, no. 1, pp. 39-45, 2008.

[37] S. Oba, M. Hino, and T. Fujita, "Adrenomedullin protects against oxidative stress-induced podocyte injury as an endogenous antioxidant," Nephrology Dialysis Transplantation, vol. 23, no. 2, pp. 510-517, 2008.

[38] K. Susztak, A. C. Raff, M. Schiffer, and E. P. Böttinger, "Glucose-induced reactive oxygen species cause apoptosis of podocytes and podocyte depletion at the onset of diabetic nephropathy," Diabetes, vol. 55, no. 1, pp. 225-233, 2006.

[39] Y. Gorin, K. Block, J. Hernandez et al., "Nox4 NAD(P)H oxidase mediates hypertrophy and fibronectin expression in the diabetic kidney," The Journal of Biological Chemistry, vol. 280, no. 47, pp. 39616-39626, 2005.

[40] S. C. Lee, S. H. Han, J. J. Li et al., "Induction of heme oxygenase-1 protects against podocyte apoptosis under diabetic conditions," Kidney International, vol. 76, no. 8, pp. 838-848, 2009.

[41] M. Brownlee, A. Cerami, and H. Vlassara, "Advanced glycosylation end products in tissue and the biochemical basis of diabetic complications," New England Journal of Medicine, vol. 318, no. 20, pp. 1315-1321, 1988.

[42] D. A. Greene, S. A. Lattimer, and A. A. F. Sima, "Sorbitol, phosphoinositides, and sodium-potassium-ATPase in the pathogenesis of diabetic complications," New England Journal of Medicine, vol. 316, no. 10, pp. 599-606, 1987.

[43] T. Nishikawa, D. Edelstein, X. L. Du et al., "Normalizing mitochondrial superoxide production blocks three pathways of hyperglycaemic damage," Nature, vol. 404, no. 6779, pp. 787790, 2000.

[44] T. Inoguchi, P. Li, F. Umeda et al., "High glucose level and free fatty acid stimulate reactive oxygen species production through protein kinase C-dependent activation of $\mathrm{NAD}(\mathrm{P}) \mathrm{H}$ oxidase in cultured vascular cells," Diabetes, vol. 49, no. 11, pp. 1939-1945, 2000.

[45] F. Forcheron, A. Basset, P. Abdallah, P. Del Carmine, N. Gadot, and M. Beylot, "Diabetic cardiomyopathy: effects of fenofibrate and metformin in an experimental model-The Zucker diabetic rat," Cardiovascular Diabetology, vol. 8, article no. 16, 2009.

[46] H. Fujii, K. Kono, K. Nakai et al., "Oxidative and nitrosative stress and progression of diabetic nephropathy in type 2 diabetes," American Journal of Nephrology, vol. 31, no. 4, pp. 342-352, 2010.

[47] M. Y. El-Mir, V. Nogueira, E. Fontaine, N. Avéret, M. Rigoulet, and X. Leverve, "Dimethylbiguanide inhibits cell respiration via an indirect effect targeted on the respiratory chain complex I," The Journal of Biological Chemistry, vol. 275, no. 1, pp. 223228, 2000.

[48] M. Foretz, S. Hébrard, J. Leclerc et al., "Metformin inhibits hepatic gluconeogenesis in mice independently of the LKB1/ AMPK pathway via a decrease in hepatic energy state," Journal of Clinical Investigation, vol. 120, no. 7, pp. 2355-2369, 2010. 
[49] T. Kiho, M. Kato, S. Usui, and K. Hirano, "Effect of buformin and metformin on formation of advanced glycation end products by methylglyoxal," Clinica Chimica Acta, vol. 358, no. 1-2, pp. 139-145, 2005.

[50] M. Zhang, Y. Dong, J. Xu et al., “Thromboxane receptor activates the AMP-activated protein kinase in vascular smooth muscle cells via hydrogen peroxide," Circulation Research, vol. 102, no. 3, pp. 328-337, 2008.

[51] D. Kukidome, T. Nishikawa, K. Sonoda et al., "Activation of AMP-activated protein kinase reduces hyperglycemia-induced mitochondrial reactive oxygen species production and promotes mitochondrial biogenesis in human umbilical vein endothelial cells," Diabetes, vol. 55, no. 1, pp. 120-127, 2006.

[52] Z. Liu, J. Li, Z. Zeng, M. Liu, and M. Wang, "The antidiabetic effects of cysteinyl metformin, a newly synthesized agent, in alloxan- and streptozocin-induced diabetic rats," ChemicoBiological Interactions, vol. 173, no. 1, pp. 68-75, 2008. 


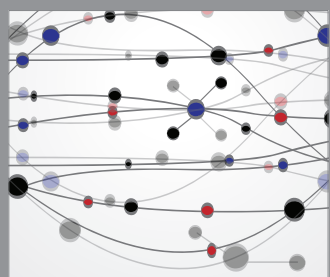

The Scientific World Journal
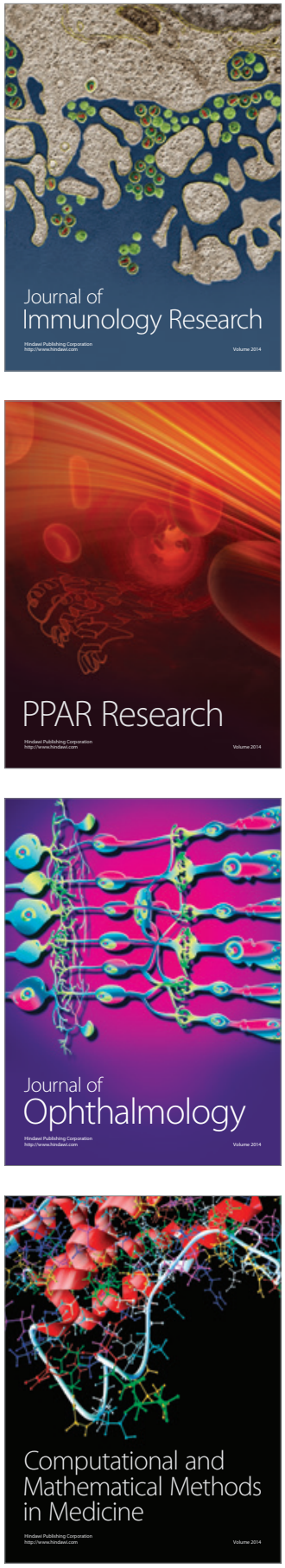

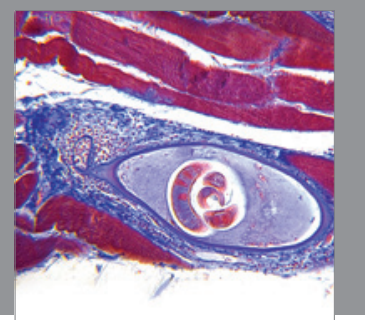

Gastroenterology

Research and Practice
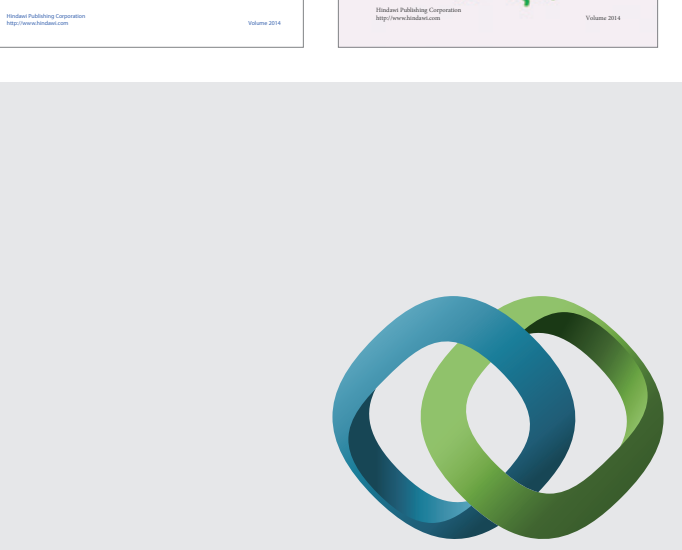

\section{Hindawi}

Submit your manuscripts at

http://www.hindawi.com
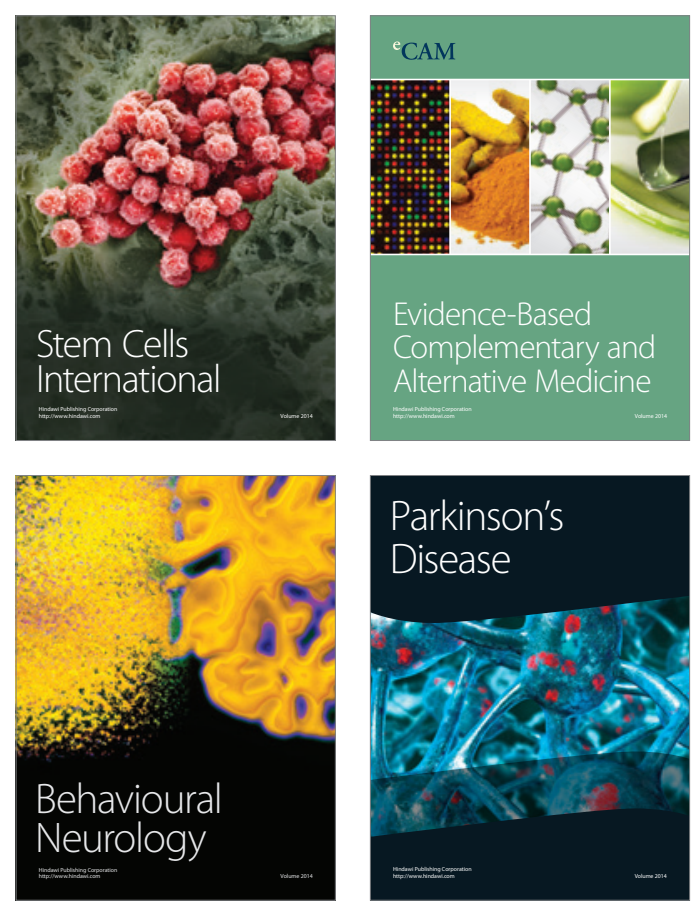

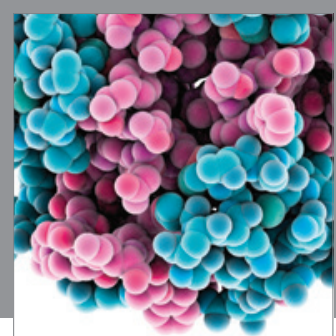

Journal of
Diabetes Research

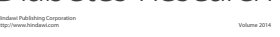

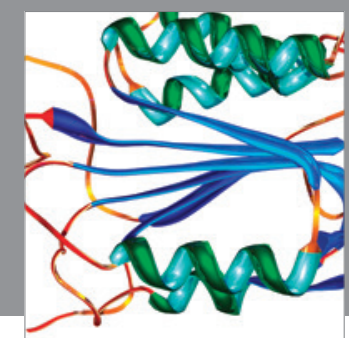

Disease Markers
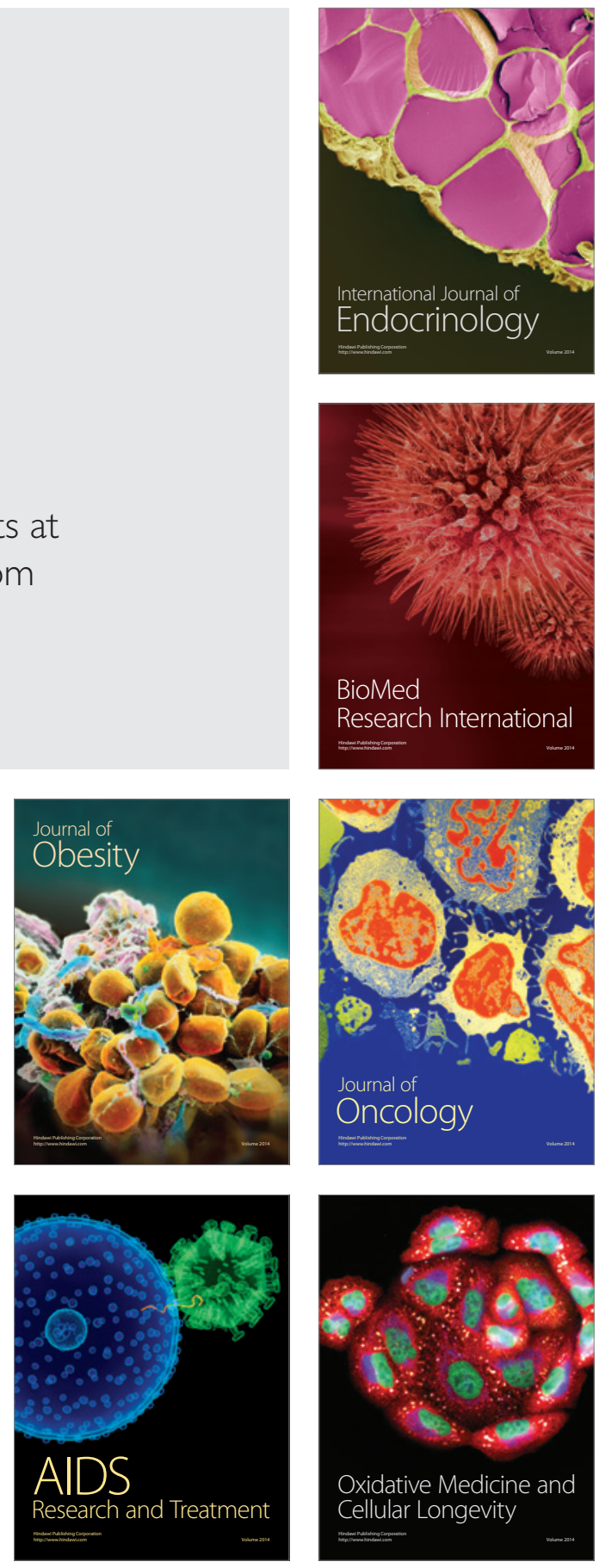